Open Access

\title{
Long-term results of chemoradiotherapy for stage II-III thoracic esophageal cancer in a single institution after 2000 -with a focus on comparison of three protocols-
}

Rei Umezawa1*, Keiichi Jingu', Haruo Matsushita', Toshiyuki Sugawara1, Masaki Kubozono', Takaya Yamamoto', Yojiro Ishikawa ${ }^{1}$, Maiko Kozumi ${ }^{1}$, Noriyoshi Takahashi ${ }^{1}$, Yu Katagiri ${ }^{1}$, Noriyuki Kadoya ${ }^{1}$, Ken Takeda ${ }^{2}$, Hisanori Ariga ${ }^{3}$, Kenji Nemoto ${ }^{4}$ and Shogo Yamada ${ }^{1}$

\begin{abstract}
Background: To evaluate the long-term results of chemoradiotherapy (CRT) for stage II-III thoracic esophageal cancer mainly by comparing results of three protocols retrospectively.

Methods: Between 2000 and 2012, 298 patients with stage II-III thoracic esophageal cancer underwent CRT. Patients in Group A received two cycles of cisplatin (CDDP) at $70 \mathrm{mg} / \mathrm{m}^{2}$ (day 1 and 29) and 5-fluorouracil (5-FU) at $700 \mathrm{mg} / \mathrm{m}^{2} / 24 \mathrm{~h}$ (day 1-4 and 29-32) with radiotherapy (RT) of 60 Gy without a break. Patients in Group B received two cycles of CDDP at $40 \mathrm{mg} / \mathrm{m}^{2}$ (day 1, 8, 36 and 43 ) and $5-\mathrm{FU}$ at $400 \mathrm{mg} / \mathrm{m}^{2} / 24 \mathrm{~h}$ (day $1-5,8-12,36-40$ and 43-47) with RT of 60 Gy with a 2-week break. Patients in Group C received two cycles of nedaplatin at $70 \mathrm{mg} / \mathrm{m}^{2}$ (day 1 and 29) and 5-FU at $500 \mathrm{mg} / \mathrm{m}^{2} / 24 \mathrm{~h}$ (day 1-4 and 29-32) with RT of 60-70 Gy without a break. Differences in prognostic factors between the groups were analyzed by univariate and multivariate analyses.

Results: The 5-year overall survival rates for patients in Group A, Group B and Group C were 52.4, 45.2 and 37.2 \%, respectively. The 5-year overall survival rates for patients in Stage II, Stage III (non-T4) and Stage III (T4) were 64.0, 40.1 and $22.5 \%$, respectively. The 5 -year overall survival rates for patients who received 1 cycle and 2 cycles of concomitant chemotherapy were 27.9 and $46.0 \%$, respectively. In univariate analysis, stage, performance status and number of concomitant chemotherapy cycles were significant prognostic factors $(p<0.001, p=0.008$ and $p<0.001$, respectively). In multivariate analysis, stage, protocol and number of concomitant chemotherapy cycles were significant factors ( $p<0.001, p=0.043$ and $p<0.001$, respectively).
\end{abstract}

Conclusions: The protocol used in Group A may be an effective protocol of CRT for esophageal cancer. It may be important to complete the scheduled concomitant chemotherapy with the appropriate intensity of CRT.

Keywords: Esophageal cancer, Stage II-III, Squamous cell carcinoma, Chemoradiotherapy

\footnotetext{
* Correspondence: reirei513@hotmail.com

'Department of Radiation Oncology, Tohoku University Graduate School of

Medicine, 1-1, Seiryou-machi, Aobaku, Sendai 980-8574, Japan

Full list of author information is available at the end of the article
} 


\section{Background}

Chemoradiotherapy (CRT) for thoracic esophageal cancer has better local control and overall survival than does radiotherapy (RT) alone and is one of the curative treatments for thoracic esophageal cancer [1]. Some studies have shown that CRT for stage I esophageal cancer had a favorable treatment outcome [2,3]. Although esophagectomy with neoadjuvant therapy has been the first choice of treatment for stage II-III, Ariga et al. and Hironaka et al. reported that treatment outcomes after CRT among patients with resectable thoracic esophageal squamous cell carcinoma were comparable to outcomes after surgery $[4,5]$. A cisplatin (CDDP)-based combination as a regimen of CRT for thoracic esophageal cancer has become the standard and was used in some clinical trials [6-9]. However, the optimal schedule and dose of chemotherapy have not been established. Moreover, because techniques for radiotherapy such as intensity modulated radiation therapy have been improved, the current outcome of CRT for thoracic esophageal cancer is expected to be better than that in past trials.

We evaluated the long-term results of CRT for stage II-III thoracic esophageal cancer after 2000 mainly by comparing results of three protocols retrospectively. We also evaluated other prognostic factors that influence the results of CRT.

\section{Methods \\ Patients}

Between 2000 and 2012, 298 patients with stage II-III (T1-4 N0-1 M0: Union for International Cancer Control 2002) thoracic esophageal cancer underwent definitive CRT. This study was performed according to the principles of the Declaration of Helsinki (2013). At the time the patients gave their consent for CRT, we did not obtain comprehensive consent including future research study. Because of retrospective study, it is difficult to reacquire agreement from the patients or their family. Therefore, information disclosure is being done to give a chance of participation refusal on home page after Tohoku University School of Medicine Institutional Review Board approved this retrospective study (2014-1-543).

\section{Radiotherapy}

Gross tumor volume was defined as the primary tumor and nodal metastasis based on upper gastrointestinal endoscopy, barium swallow, computed tomography (CT) scan and positron emission tomography (PET). If it was difficult to discriminate the primary tumor on RT planning, the clips were placed on the proximal and distal sides of the primary tumor. Initial clinical target volume (CTV) was defined as the region from the supraclavicular to celiac lymph nodes. Initial CTV was made small in consideration of the patient's general condition. Boost CTV was defined as the primary tumor with a $20-30 \mathrm{~mm}$ craniocaudal margin and an approximately $5 \mathrm{~mm}$ radial margin and nodal metastasis. Planning target volume was defined as CTV plus a 5-15 mm margin. Basically, the initial CTV received 40 Gy at 2 Gy per day using parallelopposed anterior-posterior fields. The boost CTV received 20-30 Gy at 2 Gy per day using parallel-oblique fields to avoid the spinal cord. In some cases, dose per fraction was set to $1.8 \mathrm{~Gy}$ in consideration of the patient's general condition and the size of RT fields.

\section{Protocols}

All patients underwent one of the following three protocols of CRT (Fig. 1). Adjuvant chemotherapy after CRT was performed in some patients. Patients in Group A received two cycles of chemotherapy (2-h infusion of CDDP at $70 \mathrm{mg} / \mathrm{m}^{2}$ on day 1 and continuous infusion of 5-fluorouracil [5-FU] at $700 \mathrm{mg} / \mathrm{m}^{2}$ over a 24 -h period on day 1-4) with a 4-week intervals and RT dose of $60 \mathrm{~Gy}$. This protocol has been performed since 2009. Patients in Group B received two cycles of chemotherapy (2-h infusion of CDDP at $40 \mathrm{mg} / \mathrm{m}^{2}$ on day 1 and 8 and continuous infusion of 5 -FU at $400 \mathrm{mg} / \mathrm{m}^{2}$ over a $24-\mathrm{h}$ period on day $1-5$ and 8-12) with a 4-week intervals and RT dose of 60 Gy with a 2-week break after $30 \mathrm{~Gy}$. This protocol has been performed mainly since 2000 . Patients in Group C received two cycles of chemotherapy (2-h infusion of nedaplatin [CDGP] at $70 \mathrm{mg} / \mathrm{m}^{2}$ on day 1 and continuous infusion of 5 -FU $500 \mathrm{mg} / \mathrm{m}^{2}$ over a 24-h period on day 1-5) with a 4-week interval and RT dose of 60-70 Gy. This protocol has been performed mainly since 2000. The decisions to assign patients to the three protocols was made by experienced clinicians.

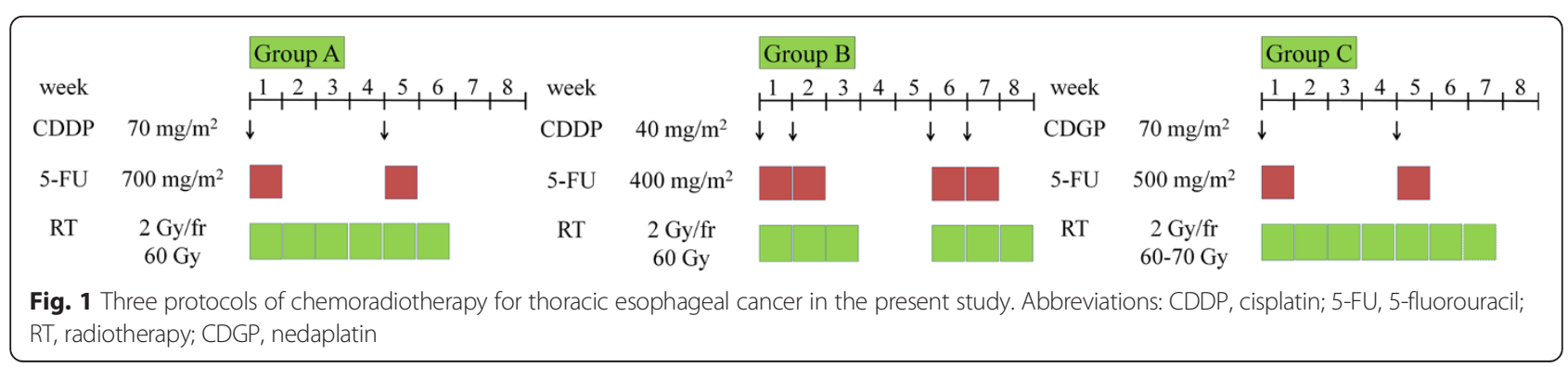




\section{Endpoints and follow-up}

The primary endpoint of the present study was the 5year overall survival rate. The secondary endpoints were progression-free survival rate, completion rate of the protocol, pattern of the first relapse and late toxicity.

Upper gastrointestinal endoscopy, CT and PET were performed for evaluation of locoregional relapse and distant metastasis every 3-6 months. We described the first treatment at the time of the first relapse.

Late toxicities were graded according to the Common Terminology Criteria for Adverse Events version 4.0. An adverse effect more than 90 days after CRT was defined as a late toxicity.

\section{Statistical analysis}

The characteristics of patients in Group A, Group B and Group $C$ were compared by the $2 \times 2$ chi-square test for dichotomous variables or the Mann-Whitney test for continuous variables. Overall survival rate and progression-free survival rate were estimated using the Kaplan-Meier method. Differences between patient subgroups for prognostic factors were analyzed using the log-rank test as univariate analysis. Overall survival was measured from the start of RT to the date of death or last follow-up. Progression-free survival was measured from the start of RT to the date of first relapse or death due to any cause. If salvage esophagectomy was performed due to a residual lesion after CRT, we made the date of salvage esophagectomy the date of relapse. Age (66 years or less vs more than 66 years), gender (male vs female), performance status (PS) ( 0 vs 1 vs 2 ), primary site (Upper thoracic esophagus vs Middle thoracic esophagus vs Lower thoracic esophagus), stage (II vs III (non-T4) vs III (T4)), protocol (Group A vs Group B vs Group C), RT dose (60 Gy or less vs more than 60 Gy), number of concomitant chemotherapy cycles ( 1 cycle vs 2 cycles), and adjuvant chemotherapy (with vs without) were included in the log-rank test. Multivariate analysis was performed using the Cox proportional hazards regression model. All tests were two-sided, and statistical significance was set at the level of $p<0.05$. Statistical analysis was performed using JMP 10 (SAS Institute Inc., Cary, NC, USA).

\section{Results}

The patients' characteristics are shown in Table 1. All patients had histologically proven squamous cell carcinoma. The numbers of patients in Group A, Group B and Group C were 48, 159 and 91, respectively. There were significant differences in age, PS, stage, RT dose, number of concomitant chemotherapy cycles and adjuvant chemotherapy between the three groups $(p<0.001, p<0.001, p=0.015, p<$ $0.001, p=0.019$ and $p<0.001$, respectively). The median ages of the patients in Group A, Group B and Group C were 67,66 and 70 years, respectively. The number of
Table 1 Patients' characteristics

\begin{tabular}{|c|c|}
\hline Characteristic & Number of patients \\
\hline \multicolumn{2}{|l|}{ Age at radiotherapy } \\
\hline 66 years or less & 140 \\
\hline More than 66 years & 158 \\
\hline \multicolumn{2}{|l|}{ Gender } \\
\hline Male & 255 \\
\hline Female & 43 \\
\hline \multicolumn{2}{|l|}{ Performance status } \\
\hline 0 & 54 \\
\hline 1 & 201 \\
\hline 2 & 27 \\
\hline Unknown & 16 \\
\hline \multicolumn{2}{|l|}{ Primary site } \\
\hline Upper thoracic esophagus & 91 \\
\hline Middle thoracic esophagus & 160 \\
\hline Lower thoracic esophagus & 47 \\
\hline \multicolumn{2}{|l|}{ Stage } \\
\hline$\|$ & 93 \\
\hline III (non-T4) & 134 \\
\hline III (T4) & 71 \\
\hline \multicolumn{2}{|l|}{ Protocol } \\
\hline Group A & 48 \\
\hline Group B & 159 \\
\hline Group C & 91 \\
\hline \multicolumn{2}{|l|}{ Radiotherapy dose } \\
\hline 60 Gy or less & 221 \\
\hline More than $60 \mathrm{~Gy}$ & 77 \\
\hline \multicolumn{2}{|l|}{ Concomitant chemotherapy } \\
\hline 1 cycle & 42 \\
\hline 2 cycles & 256 \\
\hline \multicolumn{2}{|l|}{ Adjuvant chemotherapy } \\
\hline With & 67 \\
\hline Without & 231 \\
\hline
\end{tabular}

patients with PS0/ PS1/ PS2 were 22/24/2, 17/120/10 and $15 / 57 / 15$, respectively. The numbers of patient with stage II/ stage III (non-T4)/ stage III (T4) in Group A, Group B and Group C were 17/13/18, 47/83/29 and 30/37/24, respectively.

The completion rates of RT in Group A, Group B and Group C were $100 \%$ (48/48), $95.0 \%$ (151/159) and $97.5 \%$ (89/91), respectively. Total dose at the cessation of RT was 20-64 Gy (median, 40 Gy), and a total dose of $70 \mathrm{~Gy}$ was planned in the prescription for 2 patients. The reasons for cessation of RT were brain infarct in 1 patient, myelosuppression in 2 patients, severe radiation pneumonia (Grade 5) in 2 patients, severe esophageal 
stenosis in 1 patient, esophagobronchial fistula in 1 patient, infective thrombus in 1 patient, poor general condition in 1 patient, and refusal of RT in 1 patient. The completion rates of 2 cycles of chemotherapy in Group A, Group B and Group C were $79.1 \%$ (38/48), $91.1 \%$ $(145 / 159)$ and $80.2 \%$ (73/91), respectively. In 19 patients, the dose intensity of chemotherapy in the second cycle was reduced due to myelosuppression and renal dysfunction. Adjuvant chemotherapy after CRT was performed in 67 patients. The number of cycles of adjuvant chemotherapy was $1-8$ (median, 2). The number of patients who received adjuvant chemotherapy in Group A, Group B and Group C who received adjuvant chemotherapy were $14(29.1 \%), 45(28.3 \%)$ and 8 (8.8\%), respectively.

The median follow-up period was 23.4 months (range, 1.8-150.2 months). A total of 155 patients died during the follow-up period. The 3- and 5-year survival rates in all patients were $51.5 \%$ (95\% confidence interval [CI], 45.5-57.6) and $43.5 \%$ (95\% CI, 37.4-50.0), respectively. Five patients died of second malignancy at 8.587.8 months after CRT, and 5 patients died of esophageal hemorrhage at 2.5-11.8 months after CRT. Results of the log-rank tests presented in Table 2 show the 5year overall survival rate for each prognostic factor. The 2-year overall survival rates for patients in Group A, Group B and Group C were 74.5 \% (95 \% CI, 59.4-85.5), $61.1 \%(95 \% \mathrm{CI}, 53.1-68.6)$ and $51.1 \%$ (95 \% CI, 40.461.8 ), respectively. The 5 -year overall survival rates for patients in Group A, Group B and Group C were $52.4 \%$ (95\% CI, 35.0-69.3), $45.2 \%$ (95\% CI, 37.0-53.6) and $37.2 \%$ (95 \% CI, 26.8-48.8), respectively (Fig. 2). However, there were no significant differences between the three groups $(p=0.082)$. In univariate analysis, stage, PS and number of concomitant chemotherapy cycles were significant prognostic factors $(p<0.001, p=0.008$ and $p<0.001$, respectively). The 5 -year overall survival rates for patients in stage II, stage III (non-T4) and stage III (T4) were $64.0 \%(95 \% \mathrm{CI}, 52.5-74.2)$, $40.1 \%(95 \%$ CI, 31.0-49.9) and $22.5 \%$ (95 \% CI, 13.7-35.5), respectively (Fig. 3). The 5-year overall survival rates for patients who received 1 cycle and patients who received 2 cycles of concomitant chemotherapy were $27.9 \%$ (95 \% CI, 14.5-46.9) and $46.0 \%$ (95\% CI, 39.3-52.8), respectively (Fig. 4). The 5-year overall survival rates for patients with PS0, PS1 and PS2 were 48.7 \% (95 \% CI, 33.1-64.6), 44.3 \% (95\% CI, 36.7-52.1) and $22.3 \%$ (95 \% CI, 9.5-44.1), respectively. There were no significant differences for total dose $(p=0.09)$ and adjuvant chemotherapy $(p=0.885)$. The results of multivariate analysis are shown in Table 2. Stage, protocols and number of concomitant chemotherapy cycles were significant factors $(p<0.001, p=0.043$ and $p<0.001$, respectively). The hazard ratios (HRs) for patients in stage
Table 2 Results of univariate and multivariate analyses

\begin{tabular}{|c|c|c|c|}
\hline Factor & $\begin{array}{l}\text { 5-year OS rate (\%) } \\
(95 \% \mathrm{Cl})\end{array}$ & $\begin{array}{l}\text { UA } \\
\text { ( } p \text { value) }\end{array}$ & $\begin{array}{l}\text { MA } \\
\text { ( } p \text { value) }\end{array}$ \\
\hline Age at radiotherapy & & 0.393 & 0.162 \\
\hline 66 years or less & $47.2(38.7-55.9)$ & & \\
\hline More than 66 years & $39.4(30.5-49.1)$ & & \\
\hline Gender & & 0.100 & 0.215 \\
\hline Male & $41.2(34.6-48.1)$ & & \\
\hline Female & $58.1(40.8-73.7)$ & & \\
\hline Performance status & & 0.008 & 0.655 \\
\hline 0 & $48.7(33.1-64.6)$ & & \\
\hline 1 & $44.3(36.7-52.1)$ & & \\
\hline 2 & $22.3(9.5-44.1)$ & & \\
\hline Primary site & & 0.714 & 0.810 \\
\hline Upper thoracic esophagus & $38.9(24.4-55.7)$ & & \\
\hline Middle thoracic esophagus & $42.9(34.4-51.9)$ & & \\
\hline Lower thoracic esophagus & $46.6(35.7-57.8)$ & & \\
\hline Stage & & $<0.001$ & $<0.001$ \\
\hline$\|$ & $64.0(52.5-74.2)$ & & \\
\hline III (non-T4) & $40.1(31.0-49.9)$ & & \\
\hline III (T4) & $22.5(13.7-35.5)$ & & \\
\hline Protocol & & 0.082 & 0.043 \\
\hline Group A & $52.4(35.0-69.3)$ & & \\
\hline Group B & $45.2(37.0-53.6)$ & & \\
\hline Group C & $37.2(26.8-48.8)$ & & \\
\hline Radiotherapy dose & & 0.090 & 0.973 \\
\hline 60 Gy or less & $46.0(28.1-51.2)$ & & \\
\hline More than $60 \mathrm{~Gy}$ & $39.1(38.7-53.5)$ & & \\
\hline Concomitant chemotherapy & & $<0.001$ & $<0.001$ \\
\hline 1 cycle & $27.9(14.5-46.9)$ & & \\
\hline 2 cycles & $46.0(39.3-52.8)$ & & \\
\hline Adjuvant chemotherapy & & 0.885 & 0.306 \\
\hline With & $45.1(38.1-52.5)$ & & \\
\hline Without & $38.8(26.8-52.3)$ & & \\
\hline
\end{tabular}

OS overall survival, $\mathrm{Cl}$ confidence interval, $U A$ univariate analysis, $M A$ multivariate analysis

III (non-T4) and stage III (T4) were 2.60 (95\% CI, 1.68-4.11) and 4.17 (95\% CI, 2.47-7.12), respectively. The HRs for patients in Group B and Group C were 1.99 (95\% CI, 1.11-3.78) and 2.14 (95\% CI, 1.09-4.35), respectively. The HR with 1 cycle of concomitant chemotherapy was 3.17 (95\% CI, 1.96-5.02). We investigated overall survival rates for protocols in each stage just for reference. The 5-year overall survival rates for patients in Stage II in Group A, Group B and Group C were $77.9 \%$ (95\% CI, 41.3-94.6), 68.3 \% (95 \% CI, 53.2-80.3) and $48.4 \%$ (95 \% CI, 28.7-68.7), respectively. The 5-year overall survival rates for patients in Stage III (non-T4) in 


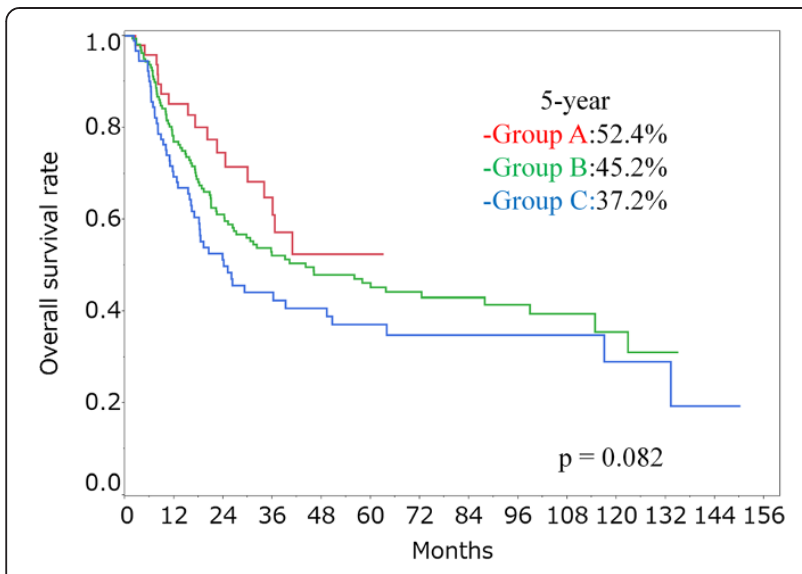

Fig. 2 Overall survival rates for Group A, Group B and Group C

Group A, Group B and Group C were 53.9 \% (95 \% CI, 25.5-80.0), $42.0 \%$ (95\% CI, 30.8-54.1) and $39.2 \%$ (95\% CI, 23.6-57.4), respectively. The 5-year overall survival rates for patients in Stage III (T4) in Group A, Group B and Group C were $25.3 \%$ (95 \% CI, 7.5-58.6), $19.1 \%$ (95 \% CI, 7.9-39.6) and $24.3 \%$ (95 \% CI, 11.0-45.4), respectively.

The 3-year and 5-year progression-free survival rates in all patients were $35.6 \%(95 \% \mathrm{CI}, 30.3-41.4)$ and $31.2 \%$ (95\%CI, 19.6-39.9), respectively. The 5-year progression-free survival rates for patients in Group A, Group B and Group C were 46.6 \% (95 \% CI, 32.3-61.6), $29.0 \%(95 \% \mathrm{CI}, 22.3-36.7)$ and $28.7 \%$ (95 \% CI, $19.6-39.9)(p=0.130)$, respectively. The 5 -year progressionfree survival rates for patients in stage II, stage III (non-T4) and stage III (T4) were $47.3 \%$ (95 \% CI, 36.7-58.1), $29.5 \%$ (95 \% CI, 22.0-38.3) and $12.8 \%$ (95 \% CI, 6.2-24.7) $(p<0.001)$, respectively. The 5 -year progression-free survival rates in patients with 1 cycle and 2 cycles of concomitant chemotherapy were $23.3 \%$ (95 \% CI, 12.6-39.1) and $32.7 \%$ (95 \% CI, 26.9-39.2) ( $p=0.003)$, respectively.

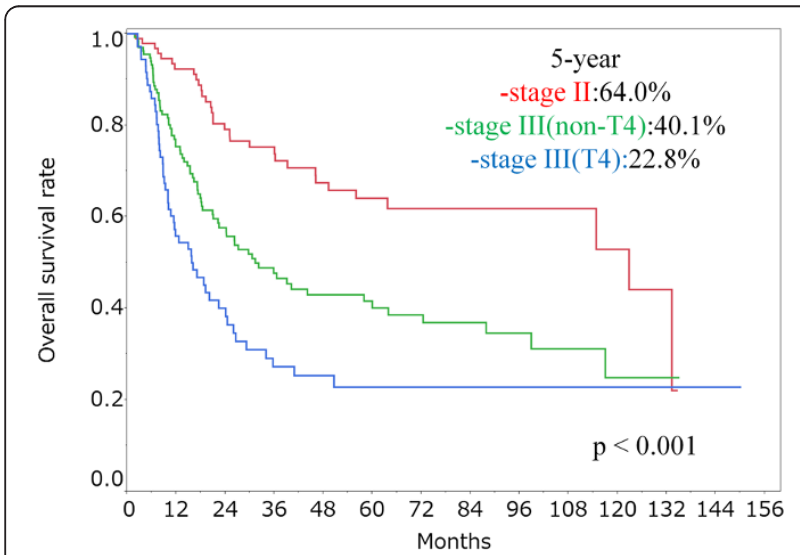

Fig. 3 Overall survival rates for patients in stage II, stage III (non-T4) and stage III (T4)

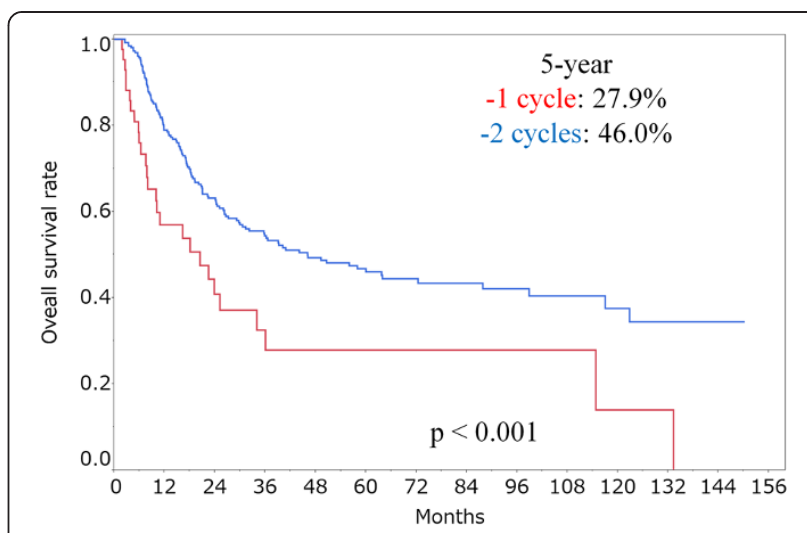

Fig. 4 Overall survival rates for patients who received 1 cycle and 2 cycles of concomitant chemotherapy

The 5-year progression-free survival rates for PS0, PS1 and PS2 were $43.1 \%$ (95 \% CI, 29.0-58.4), $29.5 \%$ (95\% CI, 23.3-36.7) and $15.9 \%$ (95\% CI, 5.6-37.3) $(p=0.032)$, respectively.

The patterns of the first relapse are shown in Table 3. One hundred seventy patients had relapse after CRT, and 109 patients had local relapse as the first relapse. Many of the first relapses occurred within one year after CRT. Salvage esophagectomy was performed in $31 \mathrm{pa-}$ tients who had local relapse and in 15 patients who had a residual local lesion. Salvage esophagectomy was performed in 16 patients in Stage II, 24 patients in Stage III (non-T4) and 6 patients in Stage III (T4) (Group A: 3 patients, Group B: 33 patients, Group C: 10 patients). The intervals from CRT to salvage esophagectomy were 1.5-29.2 months (median, 5.3 months). Two patients died of pneumonitis and gastric conduit necrosis after salvage esophagectomy. Salvage endoscopic mucosal resection or endoscopic submucosal dissection was performed in 12 patients who had local relapse. The intervals from CRT to salvage endoscopic therapy were 1.8-128.5 months (median, 14 months). Chemotherapy was performed in 55 patients as treatment for the first relapse after CRT and in 13 patients as treatment for relapse after salvage esophagectomy. Chemotherapy for locoregional relapse, distant metastasis and locoregional plus distant metastasis was performed in 34, 25 and 9 of the 68 patients, respectively (CDDP or CDGP + 5-FU: 29, CDGP + Taxane: 23, Taxane: 16). RT or CRT was

Table 3 Pattern of first relapse

\begin{tabular}{ll}
\hline Pattern of relapse & Number of patients \\
\hline Locoregional & 113 \\
$\quad$ Local relapse) & $(100)$ \\
Distant & 41 \\
Locoregional+distant & 16 \\
$\quad$ Local relapse) & (9) \\
\hline
\end{tabular}


performed in 30 patients (esophagus: 4, lymph nodes: 21, distant metastasis: 5).

Late toxicities are shown in Table 4. Two patients died of radiation pneumonitis at 5.9 months and 10.9 months after CRT. Although those patients received steroid pulse therapy, acute exacerbation was induced after that. One patient had grade 4 radiation pneumonitis. That patient recovered after steroid pulse therapy and use of a respirator. Two patients had grade 3 radiation pneumonitis. One patient died of myocardial infarction at 114.8 months after CRT, though it was not clear whether this was caused by RT. Six patients had grade 3 cardiac disorders (heart failure: 2 , acute coronary syndrome: 2 , conduction disorder: 2). Grade 3 pleural effusion and pericardial effusion were detected in 2 and 4 patients, respectively. Grade 3 esophageal stenosis or fistula was detected in 5 patients. Hypothyroidsm was detected in 6 patients and they were given levothyroxine sodium hydrate.

\section{Discussion}

We discuss the results of the present study from the point of view of esophageal squamous cell carcinoma because all patients had histologically proven squamous cell carcinoma. The 3-year and 5-year survival rates after CRT for patients with stage II-III thoracic esophageal cancer including T4 in our institution were $51.5 \%(95 \% \mathrm{CI}$, 45.5-57.6) and $43.5 \%$ (95\% CI, 37.4-50.0), respectively. The treatment results in the present study were better than those in previous studies, indicating that CRT for stage II-III thoracic esophageal cancer has been improved. Esophagectomy with neoadjuvant chemotherapy in Japan and esophagectomy with neoadjuvant CRT in Western countries have been the main treatments for stage II-III esophageal cancer as previously mentioned [10-12]. However, treatment results of CRT for stage II-III may be comparable to those of esophagectomy as shown in studies by Ariga et al. and Hironaka et al. [4, 5]. A metaanalysis of randomized trials in which definitive (chemo-) radiotherapy was compared with either surgery alone or surgery+/-induction treatment showed that

Table 4 Late toxicities

\begin{tabular}{lllll}
\hline Study & Grade 2 & Grade 3 & Grade 4 & Grade 5 \\
\hline Radiation pneumonitis & 3 & 2 & 1 & 2 \\
Pleural effusion & 13 & 2 & 0 & 0 \\
Pericardial effusion & 32 & 4 & 0 & 0 \\
Heart & 6 & 6 & 0 & 1 \\
Skin & 0 & 0 & 0 & 0 \\
Esophagus & 7 & 5 & 0 & 0 \\
Spinal cord & 0 & 0 & 0 & 0 \\
Hypothyroidism & 6 & 0 & 0 & 0 \\
\hline
\end{tabular}

overall survival rates after surgery and definitive CRT were similar, though there was a trend for more cancer-related deaths in the definitive CRT groups due to a higher risk of loco-regional progression [13]. Therefore, CRT is a reasonable treatment of thoracic esophageal cancer. However, the results of CRT for T4 esophageal cancer in the present study were poor, as shown in other studies $[14,15]$. Those results indicate the importance of early detection of esophageal cancer.

Local relapse rates after CRT were about $30 \%$ in some studies [4, 7, 16]. In the present study, 109 of the 298 patients had local relapse. Salvage esophagectomy has been the main curative treatment for local relapse after CRT, and salvage esophagectomy was performed in 46 patients in the present study. The large number of patients who received salvage esophagectomy may be the main reason for the better overall survival rate in the present study than the overall survival rates in previous studies. It is a fact that there were some patients with long-term survival after salvage esophageactomy. However, patients who underwent salvage esophagectomy after definitive high-dose CRT had high rates of morbidity and mortality [17]. Therefore, in the future, we may need to select patients having sensitivity to CRT for esophageal cancer more carefully.

Although local relapse has been a problem of CRT for esophageal cancer, increasing the complete response rate is an essential requirement to improve the results of CRT. Ishikura et al. reported that 3-year and 5-year overall survival rates were 63 and $51 \%$, respectively, for complete response patients, whereas 3-year and 5-year overall survival rates were 6 and $2 \%$, respectively, for non-complete response patients [18]. Therefore, it may be important to increase the treatment intensity of CRT to some extent. In the present study, the overall survival and progression-free survival rates in patients receiving two cycles of chemotherapy were better than those in patients receiving one cycle of chemotherapy between all of protocol groups, though we did not show those results. Therefore, it may be important to complete the scheduled protocol of CRT with the minimum of effort to reduce side effects. We compared the treatment results of three protocols in the present study. Since patients in Group B had a 2-week break after 30 Gy, the completion rate of CRT in Group B was the highest in the three groups. In contrast, the progression-free survival rate in Group B was lower than that in Group A. This might have been caused by protraction of RT. Protraction of RT has been shown to be detrimental in patients with head and neck cancer $[19,20]$. Crehange et al. also reported that local control rate of a protocol with a 2-week break during CRT was worse than that of a protocol without a break in patients with T3N0-1 esophageal cancer [21]. Therefore, we may need to avoid 
unconsidered protraction of RT for esophageal cancer. CDGP used in Group C showed anti-tumor activity similar to that of CDDP and had less renal and gastrointestinal toxicity [22]. However, the treatment results in Group $\mathrm{C}$ were worse than those in Group A. One of those reasons might be that the general conditions of patients in Group $C$ were poorer and they were older than those in Group A and Group B. Group A had the highest intensity of treatment in the three protocols and the protocol in Group A might be an effective CRT protocol for esophageal cancer, though the follow-up period was short and the number of patients in this group was small. Although a regimen consisting of CDDP + 5-FU and RT has been the standard for thoracic esophageal cancer, a variety of protocols of concomitant chemotherapy have been used. In the PRODIGE5/ACCORD17, definitive CRT with an FOLFOX treatment regimen (5-FU plus leucovorin and oxaliplatin) was compared with 5-FU and CDDP in patients with esophageal cancer [23]. In the Study of Chemoradiotherapy in OesoPhageal cancer with Erbitux (SCOPE) 1 trial, outcome of definitive CRT with or that without the addition of cetuximab to CDDP and 5-FU in patients with esophageal cancer were compared [24]. However, an improvement in overall survival was not achieved in either of the trials. Protocols that are superior to CDDP and 5-FU are expected to be established in the future.

Two cycles of adjuvant chemotherapy after concomitant CRT were performed in many prospective studies [4, 6-9]. Although adjuvant chemotherapy is often performed in patients with more advanced esophageal cancer, adjuvant chemotherapy had no significant benefit for overall survival rate or progression-free survival rate in the present study. Additional investigation of the effects of adjuvant chemotherapy may be necessary.

With respect to total RT dose, Intergroup (INT) 0123 carried out a clinical trial to compare standard dose RT (50.4 Gy) and high-dose RT (64.8 Gy) combined with CDDP and 5-FU [6]. They reported that there was no significant difference in median survival $(13.0$ vs 18.1 months) and 2-year survival (31 \% vs. $40 \%$ ) between the high-dose and standard-dose groups. Therefore, a total RT dose of 50.4 Gy has often been used in CRT for esophageal cancer, though the total RT dose in the present study was 60-70 Gy. However, the treatment results may not be the same as those in previous study because RT techniques have been improving. Treatment results have in fact been different in some studies. Suh et al. reported that high-dose radiotherapy of 60 Gy or more with concurrent chemotherapy for stage II-III patients improved locoregional control and progressionfree survival [25]. On the other hand, Kato et al. reported that the 1-year and 3-year overall survival rates after CRT at a dose of 50.4 Gy for stage II-III patients were 88.2 and $63.8 \%$, respectively, and that there were no deaths related to salvage surgery [16]. For comparison, a total dose of more than 60 Gy did not improve overall survival in the present study and might have no advantage. INT0123 also reported that 11 treatmentrelated deaths occurred in the high-dose group and only two deaths occurred in the standard-dose group [6]. That is one of the reasons why a total RT dose of 50.4 Gy has often been used in CRT for esophageal cancer in the U.S. The rates of late toxicities of Grade 3 or greater were 37 to $46 \%$ in INT0123. The rate of late toxicities was $7.7 \%$ in the present study, though those might have been underestimated because the study was a retrospective study. The rates of late toxicities in other recent studies on CRT with a total RT dose of 60 Gy were similar to that in the present study $[18,26]$. Based on those results, the appropriate total RT doses for patients with esophageal cancer who will undergo salvage esophagectomy and those who will not undergo salvage esophagectomy may be 50.4 Gy and 60 Gy, respectively.

There are some limitations in the present study. First, there were significant differences in some prognostic factors between Group A, Group B and Group C. There was no defined criteria due to the retrospective analysis in the present study. If general conditions were good in less than 80 years patients without renal, cardiac and liver dysfunction, cisplatin-based regimens such as Group A and Group B tended to be performed. Therefore, selection bias may have affected outcomes of CRT in the present study. The patients in Group C were older and the general condition of patients in Group $C$ was poorer than patients in Group A and Group B, as stated above. Second, we did not evaluate overall survival rate in view of smoking and alcohol consumption. Therefore, treatment outcomes and rate of completion of CRT might also have been affected by those factors because the patients, especially those in Group $\mathrm{C}$, might have had some comorbidities caused by those factors. Third, the median follow-up period in Group A was shorter than those in Group B and Group C because the protocol for Group A has been performed since 2009. Therefore, the evaluation of 5-year overall survival rates in the three groups might be inappropriate. However, the 2year overall survival rate in Group A was better than those in Group B and Group C, and the protocol used in Group A may therefore be an effective CRT protocol be one of for esophageal cancer.

\section{Conclusions}

CRT for stage II-III thoracic esophageal cancer is effective, and long-term survival can be expected. However, local relapse was observed in many patients. In the future, we may need to select patients having sensitivity to CRT for esophageal cancer more carefully. 
The protocol used in Group A may be an effective protocol for esophageal cancer. It may be important to complete the scheduled concomitant chemotherapy with the appropriate intensity of CRT. Additional investigation is needed to improve overall survival.

\section{Abbreviations}

CRT: Chemoradiotherapy; RT: Radiotherapy; CDDP: Cisplatin; CT: Computed tomography; PET: Positron emission tomography; CTV: Clinical target volume; 5-FU: 5-fluorouracil; CDGP: Nedaplatin; PS: Performance status; HRs: Hazard ratios; SCOPE: The Study of Chemoradiotherapy in OesoPhageal cancer with Erbitux; INT: Intergroup.

\section{Competing interests}

The authors declare that they have no competing interests.

\section{Authors' contributions}

$R U, K J, H A, K N$ and SY participated in the design of the study and reviewed the results. RU, KJ, HM, TS, MK, TY, YI, MK, NT, YK, NK and KT were responsible for the patient collection and performed radiation planning. RU, KJ, HM, NK and $\mathrm{KT}$ were responsible for the statistical analysis. $\mathrm{RU}$ drafted the manuscript. KJ, HM, KT, HA, KN and SY helped to draft the manuscript. All authors read and approved the final manuscript.

\section{Acknowledgements}

We thank all of the patients who participated in the present study and all of the personnel of the Department of Radiation Oncology for support in the present study.

\section{Author details}

'Department of Radiation Oncology, Tohoku University Graduate School of Medicine, 1-1, Seiryou-machi, Aobaku, Sendai 980-8574, Japan. ²Department of Radiological Technology, School of Health Sciences, Faculty of Medicine, Tohoku University, Sendai, Japan. ${ }^{3}$ Department of Radiology, Iwate Medical University School of Medicine, Morioka, Japan. ${ }^{4}$ Department of Radiation Oncology, Yamagata University School of Medicine, Yamagata, Japan.

\section{Received: 23 March 2015 Accepted: 19 October 2015}

Published online: 27 October 2015

\section{References}

1. Cooper JS, Guo MD, Herskovic A, Macdonald JS, Martenson Jr JA, Al-Sarraf $\mathrm{M}$, et al. Chemoradiotherapy of locally advanced esophageal cancer: longterm follow-up of a prospective randomized trial (RTOG 85-01). Radiation Therapy Oncology Group. JAMA. 1999;281(17):1623-7.

2. Jingu $K$, Matsushita $H$, Takeda $K$, Narazaki $K$, Ariga $H$, Umezawa $R$, et al. Results of chemoradiotherapy for stage I esophageal cancer in medically inoperable patients compared with results in operable patients. Dis Esophagus. 2013;26(5):522-7.

3. Kato H, Sato A, Fukuda H, Kagami Y, Udagawa H, Togo A, et al. A phase II trial of chemoradiotherapy for stage I esophageal squamous cell carcinoma: Japan Clinical Oncology Group Study (JCOG9708). Jpn J Clin Oncol. 2009;39(10):638-43.

4. Ariga H, Nemoto K, Miyazaki S, Yoshioka T, Ogawa Y, Sakayauchi T, et al. Prospective comparison of surgery alone and chemoradiotherapy with selective surgery in resectable squamous cell carcinoma of the esophagus. Int J Radiat Oncol Biol Phys. 2009;75(2):348-56.

5. Hironaka S, Ohtsu A, Boku N, Muto M, Nagashima F, Saito H, et al. Nonrandomized comparison between definitive chemoradiotherapy and radical surgery in patients with $\mathrm{T}(2-3) \mathrm{N}($ any) $\mathrm{M}(0)$ squamous cell carcinoma of the esophagus. Int J Radiat Oncol Biol Phys. 2003;57(2):425-33.

6. Minsky BD, Pajak TF, Ginsberg RJ, Pisansky TM, Martenson J, Komaki R, et al. INT 0123 (Radiation Therapy Oncology Group 94-05) phase III trial of combined-modality therapy for esophageal cancer: high-dose versus standard-dose radiation therapy. J Clin Oncol. 2002;20(5):1167-74.

7. Kato K, Muro K, Minashi K, Ohtsu A, Ishikura S, Boku N, et al. Phase II study of chemoradiotherapy with 5-fluorouracil and cisplatin for Stage I-III esophageal squamous cell carcinoma: JCOG trial (JCOG 9906). Int J Radiat Oncol Biol Phys. 2011;81(3):684-90.
8. Bedenne L, Michel P, Bouche O, Milan C, Mariette C, Conroy T, et al. Chemoradiation followed by surgery compared with chemoradiation alone in squamous cancer of the esophagus: FFCD 9102. J Clin Oncol. 2007;25(10):1160-8.

9. Nishimura Y, Hiraoka M, Koike R, Nakamatsu K, Itasaka S, Kawamura M, et al. Long-term follow-up of a randomized Phase II study of cisplatin/5-FU concurrent chemoradiotherapy for esophageal cancer (KROSG0101/JROSG021). Jpn J Clin Oncol. 2012;42(9):807-12.

10. Tepper J, Krasna MJ, Niedzwiecki D, Hollis D, Reed CE, Goldberg R, et al. Phase III trial of trimodality therapy with cisplatin, fluorouracil, radiotherapy, and surgery compared with surgery alone for esophageal cancer: CALGB 9781. J Clin Oncol. 2008;26(7):1086-92.

11. van Hagen P, Hulshof MC, van Lanschot JJ, Steyerberg EW, van Berge Henegouwen MI, Wijnhoven BP, et al. Preoperative chemoradiotherapy for esophageal or junctional cancer. N Engl J Med. 2012;366(22):2074-84.

12. Ando $\mathrm{N}$, Kato $\mathrm{H}$, Igaki $\mathrm{H}$, Shinoda $\mathrm{M}$, Ozawa $\mathrm{S}$, Shimizu H, et al. A randomized trial comparing postoperative adjuvant chemotherapy with cisplatin and 5-fluorouracil versus preoperative chemotherapy for localized advanced squamous cell carcinoma of the thoracic esophagus (JCOG9907). Ann Surg Oncol. 2012;19(1):68-74.

13. Pottgen C, Stuschke M. Radiotherapy versus surgery within multimodality protocols for esophageal cancer-a meta-analysis of the randomized trials. Cancer Treat Rev. 2012;38(6):599-604.

14. Nishimura Y, Suzuki M, Nakamatsu K, Kanamori S, Yagyu Y, Shigeoka H. Prospective trial of concurrent chemoradiotherapy with protracted infusion of 5-fluorouracil and cisplatin for T4 esophageal cancer with or without fistula. Int J Radiat Oncol Biol Phys. 2002;53(1):134-9.

15. Ishida K, Ando N, Yamamoto S, Ide H, Shinoda M. Phase II study of cisplatin and 5-fluorouracil with concurrent radiotherapy in advanced squamous cell carcinoma of the esophagus: a Japan Esophageal Oncology Group (JEOG)/Japan Clinical Oncology Group trial (JCOG9516). Jpn J Clin Oncol. 2004;34(10):615-9.

16. Kato K, Nakajima TE, Ito Y, Katada C, Ishiyama H, Tokunaga SY, et al. Phase II study of concurrent chemoradiotherapy at the dose of 50.4 Gy with elective nodal irradiation for Stage II-III esophageal carcinoma. Jpn J Clin Oncol. 2013;43(6):608-15.

17. Tachimori Y, Kanamori N, Uemura N, Hokamura N, Igaki H, Kato H. Salvage esophagectomy after high-dose chemoradiotherapy for esophageal squamous cell carcinoma. J Thorac Cardiovasc Surg. 2009;137(1):49-54.

18. Ishikura S, Nihei K, Ohtsu A, Boku N, Hironaka S, Mera K, et al. Long-term toxicity after definitive chemoradiotherapy for squamous cell carcinoma of the thoracic esophagus. J Clin Oncol. 2003;21(14):2697-702.

19. Suwinski R, Sowa A, Rutkowski T, Wydmanski J, Tarnawski R, Maciejewski B. Time factor in postoperative radiotherapy: a multivariate locoregional control analysis in 868 patients. Int J Radiat Oncol Biol Phys. 2003;56(2):399-412.

20. Maciejewski B, Withers HR, Taylor JM, Hliniak A. Dose fractionation and regeneration in radiotherapy for cancer of the oral cavity and oropharynx: tumor dose-response and repopulation. Int J Radiat Oncol Biol Phys. 1989;16(3):831-43.

21. Crehange G, Maingon P, Peignaux K, N'Guyen TD, Mirabel X, Marchal C, et al. Phase III trial of protracted compared with split-course chemoradiation for esophageal carcinoma: Federation Francophone de Cancerologie Digestive 9102. J Clin Oncol. 2007;25(31):4895-901.

22. Jingu K, Nemoto K, Matsushita H, Takahashi C, Ogawa Y, Sugawara T, et al. Results of radiation therapy combined with nedaplatin (cis-diammineglycoplatinum) and 5-fluorouracil for postoperative locoregional recurrent esophageal cancer. BMC Cancer. 2006;6:50.

23. Conroy T, Galais MP, Raoul JL, Bouche O, Gourgou-Bourgade S, Douillard JY, et al. Definitive chemoradiotherapy with FOLFOX versus fluorouracil and cisplatin in patients with oesophageal cancer (PRODIGE5/ACCORD17): final results of a randomised, phase 2/3 trial. Lancet Oncol. 2014;15(3):305-14.

24. Crosby T, Hurt CN, Falk S, Gollins S, Mukherjee S, Staffurth J, et al. Chemoradiotherapy with or without cetuximab in patients with oesophageal cancer (SCOPE1): a multicentre, phase 2/3 randomised trial. Lancet Oncol. 2013;14(7):627-37.

25. Suh YG, Lee IJ, Koom WS, Cha J, Lee JY, Kim SK, et al. High-dose versus standard-dose radiotherapy with concurrent chemotherapy in stages II-III esophageal cancer. Jpn J Clin Oncol. 2014;44(6):534-40.

26. Morota M, Gomi K, Kozuka T, Chin K, Matsuura M, Oguchi M, et al. Late toxicity after definitive concurrent chemoradiotherapy for thoracic esophageal carcinoma. Int J Radiat Oncol Biol Phys. 2009;75(1):122-8. 